\title{
Vacuum Polarization for a Massless Spin-1/2 Field in the Global Monopole Spacetime at Nonzero Temperature
}

\author{
F. C. Carvalho \\ and E. R. Bezerra de Mello* \\ Departamento de Física-CCEN \\ Universidade Federal da Paraíba \\ 58.059-970, J. Pessoa, PB \\ C. Postal 5.008 \\ Brazil
}

January 17, 2019

\begin{abstract}
In this paper we present the effects produced by the temperature in the renormalized vacuum expectation value of the zero-zero component of the energy-momentum tensor associated with massless lefthanded spinor field in the pointlike global monopole spacetime. In order to develop this calculation we had to obtain the Euclidean thermal Green function in this background. Because the expression obtained for the thermal energy density cannot be expressed in a closed form, its explicit dependence on the temperature is not completely evident. So, in order to obtain concrete information about its thermal behavior, we develop a numerical analysis of our result in the high-temperature limit for specific values of the parameter $\alpha$ which codify the presence of the monopole.
\end{abstract}

PACS numbers: 04.62.+v, 11.10.Wx, 98.80.Cq

*E-mail: emello@fisica.ufpb.br 


\section{Introduction}

The Euclidean Green function associated with a massless left-handed spin$1 / 2$ field in the pointlike global monopole spacetime, $S_{F}\left(x, x^{\prime}\right)$, was presented recently in Ref. [1]. There, $S_{F}\left(x, x^{\prime}\right)$ was obtained applying the Dirac operator $\not_{L}$, on the two-components bispinor Green function $G_{F}\left(x, x^{\prime}\right)$, which obeys a non-homogeneous second order differential equation similar to the scalar case. Using this procedure we can write

$$
S_{F}\left(x, x^{\prime}\right)=\mathcal{D}_{L} G_{F}\left(x, x^{\prime}\right) .
$$

The massless left-handed spinor thermal Green function, $S_{\beta F}\left(x, x^{\prime}\right)$, can also be obtained from the respective thermal bispinor $G_{\beta F}\left(x, x^{\prime}\right)$ in similar way. This fermionic Green function should be antiperiodic in the "Euclidean" time $\tau$ with period $\beta=1 / \kappa_{B} T, \kappa_{B}$ being the Boltzman constant and $T$ the absolute temperature.

The gravitational effect produced by a global monopole can be approximately described by a solid angle deficit in the $(3+1)$-dimensional spacetime whose line element is given by [2]

$$
d s^{2}=-d t^{2}+\frac{d r^{2}}{\alpha^{2}}+r^{2}\left(d \theta^{2}+\sin ^{2} \theta d \phi^{2}\right)
$$

where the parameter $\alpha$, which codify the presence of this object is smaller than unity. The energy-momentum tensor associated with this monopole has a diagonal form and reads: $T_{0}^{0}=T_{1}^{1}=\left(\alpha^{2}-1\right) / r^{2}$ and $T_{3}^{2}=T_{3}^{3}=0$.

Because we are interested in obtaining the thermal Green function, it is convenient to work in the Euclidean continuation of the Green function by performing a Wick rotation. So we shall work on the Euclidean version of the monopole metric above, expressed by the line element

$$
d s^{2}=d \tau^{2}+\frac{d r^{2}}{\alpha^{2}}+r^{2}\left(d \theta^{2}+\sin ^{2} \theta d \phi^{2}\right) .
$$

This paper is organized as follows. In section 2 we derive the Euclidean thermal Green function associated with a massless left-handed spin- $1 / 2$ field in the pointlike global monopole spacetime adopting the imaginary-time approach [3] and using the Schwinger-De Witt formalism. In section 3 we present a formal expression for the thermal average of the zero-zero component of the energy-momentum tensor, $\left\langle T_{00}(x)\right\rangle_{\beta}$. Because this term cannot 
be written in a closed form, its dependence on the temperature is not evident. So, in order to obtain some quantitative information about its behavior we derive more workable expressions for specific values of the parameter $\alpha$. Moreover, the complete information about its dependence on the temperature requires that we proceed a numerical evaluation of some non-analytical contributions. Here in this paper, we develop these analysis in the hightemperature regime. In section 4 we summarize our results and give our conclusion about the thermal bath of massless fermions in the global monopole spacetime. In the Appendix we derive explicitly the thermal average for the energy density for the special cases where $\alpha=1 / 2$ and give the dominant thermal contributions for the cases $\alpha=1 / 3$ and $\alpha=1 / 4$.

\section{Thermal Spinor Green Function}

The Green function associated with a massless left-handed spin-1/2 field in the global monopole spacetime has been obtained recently [1]. There this Green function is presented by applying the respective Dirac operator on the two-components bispinor as we shall summarize below.

In order to write down the Dirac operator in the spacetime described by (2) we choose the following basis tetrad:

$$
e_{(a)}^{\mu}=\left(\begin{array}{cccc}
1 & 0 & 0 & 0 \\
0 & \alpha \sin \theta \cos \varphi & \cos \theta \cos \varphi / r & -\sin \varphi / r \sin \theta \\
0 & \alpha \sin \theta \sin \varphi & \cos \theta \sin \varphi / r & \cos \varphi / r \sin \theta \\
0 & \alpha \cos \theta & -\sin \theta / r & 0
\end{array}\right)
$$

With this choice the Dirac operator for a massless left-handed fermionic field in a global monopole spacetime given by

$$
\mathcal{D}_{L}=e_{(a)}^{\mu} \sigma^{(a)}\left(\partial_{\mu}+\Gamma_{\mu}\right)
$$

reduces itself to円

$$
\mathcal{D}_{L}=i\left(\partial_{t}-\alpha \sigma^{r} \partial_{r}-\frac{1}{r} \sigma^{\theta} \partial_{\theta}-\frac{1}{r \sin \theta} \sigma^{\phi} \partial_{\phi}+\frac{1-\alpha}{r} \sigma^{r}\right),
$$

\footnotetext{
${ }^{1}$ Here $\sigma^{(a)}=\left(I, \sigma^{k}\right), \sigma^{k}$, with $k=1,2,3$, being the $2 \times 2$ Pauli matrices and $\Gamma_{\mu}$ the spin connection.
} 
with $\sigma^{u}=\vec{\sigma} \cdot \hat{\vec{u}}$, where $\hat{\vec{u}}$ denotes the standard unit vector along the three spatial directions in spherical coordinates.

The Feynman two-components propagator obeys the differential equation

$$
\mathcal{D}_{L} S_{F}\left(x, x^{\prime}\right)=\frac{1}{\sqrt{-g}} \delta^{(4)}\left(x-x^{\prime}\right) I_{(2)}
$$

and can be given in terms of the bispinor $G_{F}\left(x, x^{\prime}\right)$ by

$$
S_{F}\left(x, x^{\prime}\right)=i \mathcal{D}_{L} G_{F}\left(x, x^{\prime}\right)
$$

where $G_{F}\left(x, x^{\prime}\right)$ obeys the $2 \times 2$ differential equation

$$
\mathcal{L} G_{F}\left(x, x^{\prime}\right)=-\frac{1}{\sqrt{-g}} \delta^{(4)}\left(x-x^{\prime}\right) I_{(2)},
$$

with

$$
\mathcal{L}=\square-\frac{1}{4} R
$$

In the above equation, the generalized d'Alembertian operator is expressed by

$$
\square=g^{\mu \nu} \nabla_{\mu} \nabla_{\nu}=g^{\mu \nu}\left(\partial_{\mu} \nabla_{\nu}+\Gamma_{\mu} \nabla_{\nu}-\Gamma_{\mu \alpha}^{\alpha} \nabla_{\alpha}\right),
$$

and $R$, the scalar curvature, is equal to $2\left(1-\alpha^{2}\right) / r^{2}$ for this spacetime.

From now on we shall work with the Euclidean version of the spinor Green function. The Euclidean bispinor, $G_{E}\left(x, x^{\prime}\right)$, can also be obtained from the Schwinger-DeWitt formalism as follows:

$$
\left.G_{(} x, x\right)=\int_{0}^{\infty} d s K\left(x, x^{\prime} ; s\right)
$$

where the heat kernel, $K\left(x, x^{\prime} ; s\right)$, can be expressed in terms of the eigenfunctions of the operator $\mathcal{L}$ as

$$
K\left(x, x^{\prime} ; s\right)=\sum_{\lambda} \Phi_{\lambda}(x) \Phi_{\lambda}^{\dagger}\left(x^{\prime}\right) e^{-s \lambda^{2}}
$$

$\lambda^{2}$ being the corresponding positively defined eigenvalue. Writing

$$
\mathcal{L} \Phi(x)=-\lambda^{2} \Phi(x)
$$


the complete set of normalized solutions of the above equation is given by [国]:

$$
\begin{aligned}
\Phi_{\lambda}^{(k)}(x) & =\sqrt{\frac{\alpha p}{2 \pi r}} e^{-i E \tau} J_{\nu_{k}}(p r) \varphi_{j, m_{j}}^{(k)}, \\
\lambda^{2} & =E^{2}+\alpha^{2} p^{2}, \\
\nu_{1} & =\frac{l+1}{\alpha}-\frac{1}{2}, \nu_{2}=\frac{l}{\alpha}+\frac{1}{2},
\end{aligned}
$$

where $J_{\nu}$ is the Bessel function of the first kind and $\varphi_{j, m_{j}}^{(k)}$, with $k=1,2$, are the spinor spherical harmonics eigenfunctions of the operators $\vec{L}^{2}$ and $\sigma \cdot \vec{L}$. (The explicit expressions for these spherical harmonics and their eigenvalue equations are given in [4]. There, it is explicitly shown the dependence of $j$ with $l$.)

Now substituting (15) into (13) we get:

$$
\begin{aligned}
K_{\infty}\left(x, x^{\prime} ; s\right)= & \int_{-\infty}^{\infty} \frac{d \omega}{2 \pi} \int_{0}^{\infty} d p \sum_{l, m} \Phi_{\lambda}(x) \Phi_{\lambda}^{\dagger}\left(x^{\prime}\right) e^{-s \lambda^{2}} \\
= & \frac{1}{4 \alpha \sqrt{\pi r r^{\prime}}} \frac{1}{s^{3 / 2}} \exp ^{-\frac{(\Delta \tau)^{2} \alpha^{2}+r^{2}+r^{\prime 2}}{4 s \alpha^{2}}} \sum_{j, m_{j}}\left[I_{\nu_{1}}\left(\frac{r r^{\prime}}{2 \alpha^{2} s}\right) C_{j, m_{j}}^{(1)}\left(\Omega, \Omega^{\prime}\right)\right. \\
& \left.+I_{\nu_{2}}\left(\frac{r r^{\prime}}{2 \alpha^{2} s}\right) C_{j, m_{j}}^{(2)}\left(\Omega, \Omega^{\prime}\right)\right]
\end{aligned}
$$

where $I_{\nu}$ is the modified Bessel function and $C_{j, m_{j}}^{(k)}\left(\Omega, \Omega^{\prime}\right)=\varphi_{j, m_{j}}^{(k)}(\Omega) \varphi_{j, m_{j}}^{(k) \dagger}\left(\Omega^{\prime}\right)$. With the help of [5] we can see that for $\alpha=1, \nu_{1}=\nu_{2}=l+1 / 2$, it is possible to proceed the two summations on the quantum numbers $j$ and $m_{j}$ and (17) reduces itself to:

$$
K_{\infty}^{(\alpha=1)}\left(x, x^{\prime} ; s\right)=\frac{1}{16 \pi^{2}} \frac{e^{-\frac{\left(x-x^{\prime}\right)^{2}}{4 s}}}{s^{2}} I_{(2)} .
$$

Now we are in position to obtain the Euclidean bispinor $G_{E}\left(x, x^{\prime}\right)$ by

\footnotetext{
${ }^{2}$ In Ref. [1] we have used another parametrization for the time component. There $g_{00}=\alpha^{2}$. For this reason there is a small changing in the expressions obtained for the normalization of the field itself and its eigenvalue.
} 
using (12). Again with the help of [5] we obtain:

$$
\begin{array}{r}
G_{E}\left(x, x^{\prime}\right)=\frac{1}{2 \pi r r^{\prime}} \sum_{j, m_{j}}\left[Q_{\nu_{1}-1 / 2}(u) C_{j, m_{j}}^{(1)}\left(\Omega, \Omega^{\prime}\right)+\right. \\
\left.Q_{\nu_{2}-1 / 2}(u) C_{j, m_{j}}^{(2)}\left(\Omega, \Omega^{\prime}\right)\right],
\end{array}
$$

$Q_{\mu}$ being the Legendre function and

$$
u=\frac{\alpha^{2}\left(\tau-\tau^{\prime}\right)^{2}+r^{2}+r^{\prime 2}}{2 r r^{\prime}} .
$$

The Euclidean thermal bispinor can be obtained in the same way by using the thermal heat kernel, $K_{\beta}\left(x, x^{\prime} ; s\right)$ :

$$
G_{\beta}\left(x, x^{\prime}\right)=\int_{0}^{\infty} d s K_{\beta}\left(x, x^{\prime} ; s\right) .
$$

For an ultrastatic spacetime, i.e., static with $g_{00}=1$, Braden [6] has proved that the thermal heat kernel can be expressed in terms of the sum

$$
K_{\beta}\left(x, x^{\prime} ; s\right)=\sum_{n=-\infty}^{\infty}(-1)^{n} K_{\infty}\left(x, x^{\prime}-n \lambda \beta ; s\right),
$$

where $\lambda$ is the "Euclidean" time unit vector. The zero-temperature heat kernel, $K_{\infty}\left(x, x^{\prime} ; s\right)$, given previously by (17) can be factorized as:

$$
K_{\infty}\left(x, x^{\prime} ; s\right)=K_{(1)}\left(\tau, \tau^{\prime} ; s\right) K_{(3)}\left(\vec{x}, \vec{x}^{\prime} ; s\right),
$$

where $K_{(1)}\left(\tau, \tau^{\prime} ; s\right)$ and $K_{(3)}\left(\vec{x}, \vec{x}^{\prime} ; s\right)$ obey, respectively, the differential equations below:

$$
\left(\frac{\partial}{\partial s}-\frac{\partial^{2}}{\partial \tau^{2}}\right) K_{(1)}\left(\tau, \tau^{\prime} ; s\right)=0
$$

and

$$
\left(\frac{\partial}{\partial s}-\nabla^{i} \nabla_{i}+\frac{1}{4} R\right) K_{(3)}\left(\vec{x}, \vec{x}^{\prime} ; s\right)=0 .
$$

Identifying in (17) as the solution for (24)

$$
K_{(1)}\left(\tau, \tau^{\prime} ; s\right)=\frac{e^{-\frac{\Delta \tau^{2}}{4 s}}}{\sqrt{s}},
$$


we can see that only $K_{(1)}\left(\tau, \tau^{\prime} ; s\right)$ will be affected by the temperature, so we obtain

$$
K_{(1) \beta}\left(\tau, \tau^{\prime} ; s\right)=\frac{1}{\sqrt{s}} \sum_{n=-\infty}^{\infty}(-1)^{n} e^{-\frac{(\Delta \tau+n \beta)^{2}}{4 s}}
$$

which is antiperiodic in coordinate $\tau$. Now we can write down our result for the thermal heat kernel:

$$
\begin{aligned}
K_{\beta}\left(x, x^{\prime} ; s\right)= & \frac{1}{4 \alpha \sqrt{\pi r r^{\prime}}} \frac{1}{s^{3 / 2}} \sum_{n=-\infty}^{\infty}(-1)^{n} \exp ^{-\frac{(\Delta \tau+n \beta)^{2} \alpha^{2}+r^{2}+r^{\prime 2}}{4 s \alpha^{2}}} \\
& \sum_{j, m_{j}}\left[I_{\nu_{1}}\left(\frac{r r^{\prime}}{2 \alpha^{2} s}\right) C_{j, m_{j}}^{(1)}\left(\Omega, \Omega^{\prime}\right)+I_{\nu_{2}}\left(\frac{r r^{\prime}}{2 \alpha^{2} s}\right) C_{j, m_{j}}^{(2)}\left(\Omega, \Omega^{\prime}\right)\right]
\end{aligned}
$$

Finally substituting (28) into (21), we obtain an explicit expression for the thermal bispinor, $G_{\beta}\left(x, x^{\prime}\right)$ :

$$
\begin{aligned}
G_{\beta}\left(x, x^{\prime}\right)= & \frac{1}{2 \pi r r^{\prime}} \sum_{n=-\infty}^{\infty}(-1)^{n} \sum_{j, m_{j}}\left[Q_{\nu_{1}-1 / 2}\left(u_{n \beta}\right) C_{j, m_{j}}^{(1)}\left(\Omega, \Omega^{\prime}\right)\right. \\
& \left.+Q_{\nu_{2}-1 / 2}\left(u_{n \beta}\right) C_{j, m_{j}}^{(2)}\left(\Omega, \Omega^{\prime}\right)\right]
\end{aligned}
$$

where

$$
u_{n \beta}=\frac{\alpha^{2}(\Delta \tau+n \beta)^{2}+r^{2}+r^{\prime 2}}{2 r r^{\prime}} .
$$

As we can see form (29), the thermal bispinor contains explicitly the zerotemperature Green function, $G_{E}\left(x, x^{\prime}\right)$, plus purely thermal contributions. Moreover, because the Legendre functions, $Q_{\mu}(z)$, decrease proportionally with $1 / z^{\mu+1}$ for large arguments [5], we conclude that, for a given set of values of the quantum numbers $j$ and $m_{j}$, the most relevant thermal corrections to $G_{\beta}\left(x, x^{\prime}\right)$ above, come from the first terms in the series in $n$.

Finally we can write down our Euclidean thermal spinor Green function substituting (29) and the Euclidean version of (6) into (8):

$$
S_{\beta}\left(x, x^{\prime}\right)=i\left(-i \partial_{\tau}-\alpha \sigma^{(r)} \partial_{r}+\frac{1}{r} \sigma^{(r)} \vec{\sigma} \cdot \vec{L}+\frac{1-\alpha}{r} \sigma^{r}\right) G_{\beta}\left(x, x^{\prime}\right) .
$$




\section{Thermal Average of the Energy-Momentum Tensor}

The energy-momentum tensor associated with spin- $1 / 2$ fields, $T_{\mu \nu}(x)$, is bilinear function of the fields. Consequently its vacuum expectation value (VEV), $\left\langle T_{\mu \nu}(x)\right\rangle$, can be evaluated by the standard method using the Green function [0].

In [1] the renormalized VEV of the energy-momentum tensor associated with a left-handed spin-1/2 field in a global monopole spacetime was explicitly calculated. There, it was shown that it has the following structure:

$$
\left\langle T_{\mu}^{\nu}(x)\right\rangle^{R e g .}=\frac{1}{8 \pi^{2} r^{4}}\left[A_{\mu}^{\nu}+B_{\mu}^{\nu} \ln (\mu r)\right],
$$

where the scaling parameter $\mu$ appears after the renormalization procedure. In fact, applying the point-splitting renormalization procedure and extracting from the spinor Green function all the divergences by subtracting the correspondent Hadamard function, the renormalized VEV of the energymomentum tensor presents a scale-dependent logarithmic term. This is a consequence of an ambiguity in the definition of $\left\langle T_{\mu \nu}\right\rangle^{\text {Reg. }}$ in a even dimensional curved spacetime. The back-reaction probelm consists of solving the Einstein equaion in presence of quantum corrections. So, this one-loop equation contains besides $\left\langle T_{\mu \nu}\right\rangle^{R e g}$. as source of energy-momentum tensor, extra purely geometrical terms [7]. The coefficients of these terms must depend on the renormalization scale since the theory must be independent of this scale due to renormalization group equation. The tensors $A_{\mu}^{\nu}$ and $B_{\mu}^{\nu}$ depend only on the metric parameter $\alpha$. Moreover, because of the spherical symmetry these tensors are diagonal with components $A_{\theta}^{\theta}=A_{\phi}^{\phi}$ and $B_{\theta}^{\theta}=B_{\phi}^{\phi}$. Therefore there are only six unknown components. The renormalized VEV of the energy-momentum tensor must be conserved, i.e.,

$$
\left\langle T_{\mu}^{\nu}\right\rangle_{; \nu}^{\text {Reg. }}=0
$$

and gives the correct conformal anomaly [8], which for massless spinor twocomponents field, reads [9]

$$
\left\langle T_{\mu}^{\mu}\right\rangle^{\text {Reg. }}=\frac{1}{16 \pi^{2}} \operatorname{tra}_{2}=\frac{\mathcal{T}}{8 \pi^{2} r^{4}} .
$$


Here we have introduced the new definition $\mathcal{T}:=\frac{r^{4} t r a_{2}}{2}$. The general form of the coefficient $a_{2}$ may be found in [10], which for the global monopole spacetime has the following form:

$$
a_{2}=-\frac{1-\alpha^{4}}{60 r^{4}} I_{(2)} .
$$

Taking into account (33) and (34) it is possible to express the tensors $A_{\mu}^{\nu}$ and $B_{\mu}^{\nu}$ in terms of their zero-zero components and the trace $T$ by

$$
A_{\mu}^{\nu}=\operatorname{diag}\left(A_{0}^{0},-\mathcal{T}+A_{0}^{0}+B_{0}^{0}, \mathcal{T}-A_{0}^{0}-\frac{B_{0}^{0}}{2}, \mathcal{T}-A_{0}^{0}-\frac{B_{0}^{0}}{2}\right),
$$

and

$$
B_{\mu}^{\nu}=B_{0}^{0} \operatorname{diag}(1,1,-1,-1) .
$$

So the only problem left is to determine the components $A_{0}^{0}$ and $B_{0}^{0}$. Using the point-splitting approach, the VEV of the energy-momentum tensor for a massless spinor field is given by:

$$
\left\langle T_{\mu \nu}(x)\right\rangle=\frac{1}{4} \lim _{x^{\prime} \rightarrow x} \operatorname{tr}\left[\sigma_{\mu}\left(\nabla_{\nu}-\nabla_{\nu^{\prime}}\right)+\sigma_{\nu}\left(\nabla_{\mu}-\nabla_{\mu^{\prime}}\right)\right] S_{F}\left(x, x^{\prime}\right),
$$

by which we have

$$
\left\langle T_{00}(x)\right\rangle=i \lim _{x^{\prime} \rightarrow x} \partial_{t}^{2} \operatorname{tr}\left(G\left(x, x^{\prime}\right)\right)=-\lim _{x^{\prime} \rightarrow x} \partial_{\tau}^{2} \operatorname{tr}\left(G_{E}\left(x, x^{\prime}\right)\right) .
$$

It was pointed out in [1], that only the time derivative in the bispinor gives a nonzero contribution to the zero-zero component of the energy-momentum tensor. Moreover taking the coincidence limit $\Omega=\Omega^{\prime}$ into (19), summing over $m_{j}$ and using the integral representation for the Legendre function [5], it is possible to develop the sum over $j$. Finally we arrive at the following expression for the Euclidean bispinor:

$$
G_{E}\left(\Delta \tau, r, r^{\prime}\right)=\frac{1}{16 \pi^{2} r r^{\prime}} \int_{b}^{\infty} \frac{d x}{\sqrt{x^{2}-b^{2}}} \frac{1}{\sinh ^{2}\left(\frac{\operatorname{arcsinh}(\mathrm{x})}{\alpha}\right)} I_{(2)},
$$

where the function $b$ is expressed in terms of the one-half of the square of the geodesic distance $\sigma$ in $(\tau, r)$ surface

$$
b^{2}=\frac{\alpha^{2}}{2 r r^{\prime}} \sigma
$$


with

$$
2 \sigma=\Delta \tau^{2}+\frac{\Delta r^{2}}{\alpha^{2}}
$$

The Green function (40) is divergent in the coincidence limit $b \rightarrow 0$. So, in order to obtain a finite and well defined result for (39), we must renormalize it subtracting all its divergences. There, it was adopted the pointsplitting renormalization procedure and subtracted from the Green function the Hadamard one, $G_{H}\left(x, x^{\prime}\right)$, which is expressed in terms of the square of geodesic distance $\sigma$. The latter presents short distance behaviors proportional to $1 / \sigma$, as a free theory, plus a logarithmic scale-dependent term proportional to the scalar curvature of the spacetime $R \ln \left(\mu^{2} \sigma\right)$.

The thermal average of the energy-momentum tensor of a thermal bath of massless fermions in the global monopole spacetime can be computed in similar way replacing the ordinary spinor Greens function in (38) by its thermal correspondent, $S_{\beta}\left(x, x^{\prime}\right)$, given in (31). For the sake of simplicity let us consider in this paper the zero-zero component of the thermal average of the energy-momentum tensor, which is also given by

$$
\left\langle T_{00}(x)\right\rangle_{\beta}=-\lim _{x^{\prime} \rightarrow x} \partial_{\tau}^{2} \operatorname{tr}\left(G_{\beta}\left(x, x^{\prime}\right)\right)
$$

The above expression gives us a divergent result which comes exclusively from the the zero-temperature contribution of the thermal bispinor, the component $n=0$ of (29), which we denominate by $G_{\infty}\left(x, x^{\prime}\right)$. In fact this singularity is consequence of the evaluation of the Legendre function at unity. So in order to obtain a finite and well defined result for (43) we have to renormalize its zero-temperature part only, $\left\langle T_{00}(x)\right\rangle_{\infty}$. In this way the renormalized thermal average of the zero-zero component of the energy-momentum tensor can be expressed by

$$
\left\langle T_{00}(x)\right\rangle_{\beta}^{\text {Reg. }}=\left\langle T_{00}(x)\right\rangle_{\infty}^{\text {Reg. }}+\left\langle\bar{T}_{00}(x)\right\rangle_{\beta}
$$

Because the first term in the right hand side of the above equation has been computed before, in this paper we calculate the purely thermal correction, $\left\langle\bar{T}_{00}(x)\right\rangle_{\beta}$. Again taking the coincidence limit $\Omega=\Omega^{\prime}$ into (29) and repeating the same procedure adopted for the zero-temperature case, we get

$$
\left\langle\bar{T}_{00}(x)\right\rangle_{\beta}=-\lim _{\Delta \tau \rightarrow 0} \partial_{\tau}^{2} \operatorname{tr}\left(\bar{G}_{\beta}(\Delta \tau, r)\right),
$$


where

$$
\bar{G}_{\beta}(\Delta \tau, r)=\frac{1}{16 \pi^{2} r^{2}} \sum_{n \neq 0}(-1)^{n} \int_{b_{n}}^{\infty} \frac{d x}{\sqrt{x^{2}-b_{n}^{2}}} \frac{1}{\sinh ^{2}\left(\frac{\operatorname{arcsinh}(\mathrm{x})}{\alpha}\right)} I_{(2)},
$$

being $b_{n}^{2}=\frac{\alpha^{2}(\Delta \tau+n \beta)^{2}}{4 r^{2}}$. Because $b_{n}>0$, the purely thermal correction in (44) is finite. Changing the variable $x$ by $b_{n} x$ in (46), taking its second time derivative followed by the coincidence limit, we find:

$$
\begin{aligned}
\left\langle\bar{T}_{00}(x)\right\rangle= & -\frac{1}{8 \pi^{2} r^{4}} \sum_{n=1}^{\infty}(-1)^{n} \int_{1}^{\infty} \frac{d z z^{2}}{\sqrt{z^{2}-1}}\left[\frac{2 \cosh ^{2}(y)+1}{\sinh ^{4}(y)\left(1+\frac{z^{2} \alpha^{2} n^{2} \beta^{2}}{4 r^{2}}\right)}\right. \\
& \left.-\frac{z \alpha^{2} n \beta}{2 r} \frac{\cosh (y)}{\sinh ^{3}(y)} \frac{1}{\left(1+\frac{z^{2} \alpha^{2} n^{2} \beta^{2}}{4 r^{2}}\right)^{3 / 2}}\right],
\end{aligned}
$$

with

$$
y=\frac{\operatorname{arcsinh}\left(\frac{\mathrm{z} \alpha \mathrm{n} \beta}{2 \mathrm{r}}\right)}{\alpha} .
$$

Unfortunately, from the above expression, it is not possible to obtain any information about the behavior of the energy density associated with a thermal bath of massless fermions in this spacetime as a function of the temperature. At this stage we only can observe that the integrand decreases exponentially for large values of $z$ and that the series in $n$ converge faster than $1 / n^{2}$ when $n$ goes to infinity. Because we want to have a concrete information about the dependence of $\left\langle T_{00}\right\rangle_{\beta}$ with the temperature, our next steps is to present this dependence for some specific values of the parameter $\alpha$. In what follows we present this information for three different situations: a) $\alpha$ close to unity, small solid angle deficit (excess), b) small value of $\alpha$, large solid angle deficit and $c$ ) large value of $\alpha$, large solid angle excess. (In the Appendix we also present some concrete results for $\alpha=1 / 2$ and brief discussion for the cases $\alpha=1 / 2$ and $\alpha=1 / 4$.)

\section{3..1 Parameter $\alpha$ close to the unity.}

In this subsection we present the explicit dependence of the purely thermal correction to $\left\langle\bar{T}_{00}(x)\right\rangle_{\beta}$ admitting that $|1-\alpha|<<1$. In fact, because the global monopole is a very heavy object which appears as consequence of a 
breakdown global $O(3)$ gauge symmetry to $U(1)$, for a typical grand unified theory $1-\alpha^{2} \sim 10^{-5}$. So in this case we can expand (47) in powers of the parameter $\eta^{2}=1-\alpha^{2}$. After long calculation we arrive, up to the first order in $\eta^{2}$, at:

$$
\begin{aligned}
\left\langle\bar{T}_{00}(x)\right\rangle_{\beta}= & \frac{7 \pi^{2}}{120 \beta^{4}}\left(1+2 \eta^{2}\right)+\frac{\eta^{2}}{4 \pi^{2} r^{4}} \sum_{n=1}^{\infty} \frac{(-1)^{n}}{n^{2}} \frac{\operatorname{arctanh}\left(\frac{1}{\sqrt{1+\mathrm{n}^{2} \beta^{2} / 4 \mathrm{r}^{2}}}\right)}{(\beta / r)^{2} \sqrt{1+n^{2} \beta^{2} / 4 r^{2}}} \\
& +\frac{2 \eta^{2}}{\pi^{2} r^{4}} \sum_{n=1}^{\infty} \frac{(-1)^{n}}{n^{5}} \frac{1}{(\beta / r)^{5}} \int_{1}^{\infty} \frac{d z}{\sqrt{z^{2}-1}} \frac{1}{z^{3}} \operatorname{arcsinh}(\mathrm{zn} \beta / 2 \mathrm{r}) \times \\
& \frac{\left[6\left(\frac{z n \beta}{2 r}\right)^{4}+19\left(\frac{z n \beta}{2 r}\right)^{2}+12\right]}{\left(1+z^{2} n^{2} \beta^{2} / 4 r^{2}\right)^{3 / 2}} .
\end{aligned}
$$

Again this result also does not clarify completely our objective because of the presence of two contributions which cannot be expressed in terms any functions found in literature. However the above expression has some advantage when compared with the previous one obtained without approximation, Eqs. (47) and (48). From (49) it is possible to proceed a numerical evaluation of both contributions for some specific values of the ratio $\xi:=\beta / r$ and in this way to provide a quantitative information for $\left\langle\bar{T}_{00}\right\rangle_{\beta}$. Our numerical analysis were developed in the high temperature or large distance limit, $\xi<<1$, and the results are shown in Figs. $1(a)$ and $1(b)$ for $S_{1}(\xi)$ and $S_{2}(\xi)$, representing, respectively, the first and second summation as shown below:

$$
S_{1}(\xi)=\sum_{n=1}^{\infty} \frac{(-1)^{n}}{n^{2}} \frac{1}{\xi^{2}} \frac{\operatorname{arctanh}\left(\frac{1}{\sqrt{1+\mathrm{n}^{2} \xi^{2} / 4}}\right)}{\sqrt{1+n^{2} \xi^{2} / 4}}
$$

and

$$
\begin{aligned}
S_{2}(\xi)= & \sum_{n=1}^{\infty} \frac{(-1)^{n}}{n^{5}} \frac{1}{\xi^{5}} \int_{1}^{\infty} \frac{d z}{\sqrt{z^{2}-1}} \frac{1}{z^{3}} \operatorname{arcsinh}(\mathrm{zn} \xi / 2) \times \\
& \frac{\left[6\left(\frac{z n \xi}{2}\right)^{4}+19\left(\frac{z n \xi}{2}\right)^{2}+12\right]}{\left(1+z^{2} n^{2} \xi^{2} / 4\right)^{3 / 2}} .
\end{aligned}
$$

From the graphs displayed in Fig. 2, which exhibits the logarithmic behavior of the previous summations, it is possible to infer the following dependence 
for $S_{1}$ and $S_{2}$ with $\xi$ :

$$
S_{1}(\xi)=\frac{a_{1}}{\xi^{q_{1}}}
$$

and

$$
S_{2}(\xi)=\frac{a_{2}}{\xi^{q_{2}}}
$$

where $a_{1}=-4.701$ with $q_{1}=2.228 \pm 0.029$ and $a_{2}=-8.735$ with $q_{2}=4.000 \pm 0.000$. From these two results we can see that the most relevant contribution comes from $S_{2}$, which, according to the precision of our numerical analysis, provides to (49) a term proportional to $1 / \beta^{4}$, so independent of the distance from the point to the global monopole.

\section{3..2 Small Parameter: $\alpha<<1$}

In the limit $\alpha<<1$ the leading term in the expansion of the integrand of (47) give us

$$
\left\langle\bar{T}_{00}(x)\right\rangle_{\beta}=-\frac{1}{8 \pi^{2} r^{4}} \sum_{n=1}^{\infty}(-1)^{n} \int_{1}^{\infty} d z \frac{z^{2}}{\sqrt{z^{2}-1}} \frac{2 \cosh ^{2}(z n \beta / 2 r)+1}{\sinh ^{4}(z n \beta / 2 r)},
$$

which becomes independent on the parameter $\alpha$. Once more this result does not elucidate our main question, which is to provide the information about the dependence of the thermal correction $\left\langle\bar{T}_{00}(x)\right\rangle_{\beta}$ with the temperature. Again only numerical evaluation provides this information. The summation part, denominate by $S_{3}$, is clearly a function of the ratio $\xi=\beta / r$. Again our numerical results in the high temperature regime, $\xi<<1$, are exhibited in Fig. 3(a) for $S_{3}$ itself and in Fig. 3(b) for its logarithmic. From both it is possible to conclude that

$$
S_{3}(\xi)=\frac{a_{3}}{\xi^{q_{3}}},
$$

where $a_{3}=-9.638$ with $q_{3}=4.000 \pm 0.000$. This result indicates that for large solid angle deficit, the high-temperature contribution to the energy density is uniform and independent on the parameter $\alpha$, i.e., $\left\langle T_{00}\right\rangle_{\beta} \sim 1 / \beta^{4}$. 


\section{3..3 Large Parameter: $\alpha>>1$}

Now in the limit $\alpha>>1$, the leading term in the expansion of (47) reads:

$$
\begin{aligned}
\left\langle\bar{T}_{00}(x)\right\rangle_{\beta}= & -\frac{\alpha^{2}}{2 \pi^{2} r^{2} \beta^{2}}\left[3 \sum_{n=1}^{\infty} \frac{(-1)^{n}}{n^{2}} \int_{1}^{\infty} \frac{d z}{\sqrt{z^{2}-1}} \frac{1}{[\ln (\alpha z n \beta / r)]^{4}}\right. \\
& \left.+\sum_{n=1}^{\infty} \frac{(-1)^{n}}{n^{2}} \int_{1}^{\infty} \frac{d z}{\sqrt{z^{2}-1}} \frac{1}{[\ln (\alpha z n \beta / r)]^{3}}\right]
\end{aligned}
$$

The above result presents two terms whose their sum cannot be evaluated analytically. They appear as the first terms of an expansion of a well defined expression. Consequently they are valid only in the case where the argument of the logarithmic never becomes equal to the unity. This means that, from the numerical point of view, $\alpha$ should assume values bigger than some critical one, which depends on the interval domain of the variable $\xi$, in such way the divergence is never attained. In order to proceed a numerical evaluation of both terms, let us write down both terms in a convenient form, absorbing the factor $r^{2} / \beta^{2}=1 / \xi^{2}$. Defining them by $S_{4}$ and $S_{5}$ they are given as:

$$
S_{4}(\xi)=\sum_{n=1}^{\infty} \frac{(-1)^{n}}{n^{2}} \frac{1}{\xi^{2}} \int_{1}^{\infty} \frac{d z}{\sqrt{z^{2}-1}} \frac{1}{[\ln (\alpha z n \xi)]^{4}}
$$

and

$$
S_{5}(\xi)=\sum_{n=1}^{\infty} \frac{(-1)^{n}}{n^{2}} \frac{1}{\xi^{2}} \int_{1}^{\infty} \frac{d z}{\sqrt{z^{2}-1}} \frac{1}{[\ln (\alpha z n \xi)]^{3}} .
$$

In this way there appears the factor $1 / r^{4}$ multiplying both terms in (56). Adopting for $\xi=\beta / r$ values in the same interval used in the other previous numerical analysis our results for the two summations can be expressed as shown below:

$$
S_{4}(\xi)=\frac{a_{4}}{\xi^{q_{4}}}
$$

and

$$
S_{5}(\xi)=\frac{a_{5}}{\xi^{q_{5}}},
$$

where we exhibit in Tables 1 and 2 the exponents and respective coefficients for different values of $\alpha$ for $S_{4}$ and $S_{5}$, respectively. Unfortunately we could not find any relationship between these parameters with $\alpha$, although we can notice no significant variations on them, i.e., the exponents, $q_{4}$ and $q_{5}$, do not change appreciably when we change $\alpha$. The dominant dependence of $\left\langle\bar{T}_{00}\right\rangle_{\beta}$ with $\alpha$ is explicitly given by the power $\alpha^{2}$ in (56). 


\section{Concluding Remarks}

In this paper we have considered the thermal average of the energy density associated with a thermal bath of massless spin- $1 / 2$ fermions in the background of a pointlike global monopole. The reason why we have taken into account massless field is because in this case it is possible to express the spinor Green function in a closed form in terms of special functions. Moreover, we decided to consider only one helicity state, the left-handed one, associated with the neutrino's degrees of freedom. In order to develop this analysis we have derived a general expression for the respective Euclidean thermal spinor Green function, $S_{\beta}\left(x, x^{\prime}\right)$. Using this Green function we obtained a formal expression for the the zero-zero component of the thermal average of the energy-momentum tensor, $\left\langle T_{00}\right\rangle_{\beta}$, which contains a zero temperature term plus purely thermal correction. Because this correction is expressed in terms of not solvable integrals, its dependence on the temperature could not be evaluated explicitly. So, to get some quantitative information, we decided to analyze this term for specific values of the parameter $\alpha$. In this way three formal expressions were obtained for special cases related in last section. Unfortunately our results were expressed in terms of infinite series not analytically evaluated; however from them it is possible to develop a numerical analysis. We developed these analysis in a high-temperature regime. Our final results enables us to evaluate qualitatively the thermal corrections, $\left\langle\bar{T}_{00}\right\rangle_{\beta}$. For the cases $\alpha$ close and much smaller than unity, the corrections are dominated by terms of order $T^{4}$, consequently independent of the distance from the point to the monopole. For the case $\alpha>>1$, there appears an explicit dependence of this correction with the distance.

The obtained results may have some applications in the early cosmology, where the temperature of the Universe was really high. We can see that the thermal contributions modify significantly the zero-temperature energy density in that epoch.

A few years ago, Linet [11] has obtained the Euclidean spinor thermal Green function for a massless spin-1/2 fermionic field in the spacetime of a global cosmic string. There, it was possible to express the thermal average of the energy-momentum tensor in a closed form. Linet has found that the high-temperature dominant contribution for energy density is proportional to $T^{4}$ and that the thermal bath of massless fermions is not perturbed by 
the presence of the straight cosmic string ${ }^{3}$. In the pointlike global monopole spacetime we cannot say that. For two specific cases treated here the results for thermal energy-densities in the high-temperature regime present explicit dependence on the parameter $\alpha$. Only the case where $\alpha<<1$ does not present this dependence.

The contribution due to the temperature in the renormalized vacuum expectation value of the square of the massless scalar field and its respective energy density in the global monopole spacetime, were recently calculated in [12] using similar procedure adopted in this paper. There appears a nonvanishing contribution to the energy density, coming from the geometry of the spacetime, to the dominant $1 / \beta^{4}$ term.

\section{A Thermal-Energy Density for Specific Val- ues of $\alpha$}

Here in this appendix we present the purely thermal correction for the energy density for the cases where the parameter $\alpha$ assume very specific values. In this first part we take $\alpha=1 / 2$. For this case it is possible to develop the integral which appears in (46). It is given by

$$
\bar{G}_{\beta}(\Delta \tau, R)=\frac{1}{64 \pi^{2} r^{2}} \sum_{n \neq 0}(-1)^{n}\left[\frac{1}{b_{n}^{2}}-\frac{1}{\sqrt{1+b_{n}^{2}}} \operatorname{arctanh}\left(\frac{1}{\sqrt{1+\mathrm{b}_{\mathrm{n}}^{2}}}\right)\right],
$$

with $b_{n}=\frac{\Delta \tau+n \beta}{4 r}$. From the above expression we get, after some calculations, the following result:

$$
\begin{aligned}
\left\langle\bar{T}_{00}(x)\right\rangle_{\beta}= & \frac{7 \pi^{2}}{120 \beta^{4}}-\frac{1}{256 \pi^{2} r^{4}}-\frac{1}{192 \beta^{2} r^{2}}+\frac{1}{256 \pi \beta r^{3}} \operatorname{cossech}\left(\frac{4 \pi \mathrm{r}}{\beta}\right) \\
& +\frac{3}{64 \beta^{2} r^{2}} \cosh \left(\frac{4 \pi r}{\beta}\right) \operatorname{cossech}^{2}\left(\frac{4 \pi \mathrm{r}}{\beta}\right)
\end{aligned}
$$

\footnotetext{
${ }^{3}$ The high-temperature dominant contribution given in the Linet's paper, $\left\langle\bar{T}_{00}(x)\right\rangle=$ $\frac{7 \pi^{2}}{60 \beta^{4}}$, is precisely twice bigger than the flat one found in our result in $(49)$. The discrepancy between both results is because we considered in this paper just one helicity state for the fermionic field.
} 


$$
\begin{aligned}
& +\frac{1}{128 \pi^{2} r^{4}} \sum_{n=1}^{\infty}(-1)^{n} \frac{\operatorname{arctanh}\left(\frac{1}{\sqrt{1+\mathrm{n}^{2} \beta^{2} / 16 \mathrm{r}^{2}}}\right)}{\left(1+n^{2} \beta^{2} / 16 r^{2}\right)^{3 / 2}} \\
& -\frac{3}{256 \pi^{2} r^{4}} \sum_{n=1}^{\infty}(-1)^{n} \frac{\operatorname{arctanh}\left(\frac{1}{\sqrt{1+\mathrm{n}^{2} \beta^{2} / 16 \mathrm{r}^{2}}}\right)}{\left(1+n^{2} \beta^{2} / 16 r^{2}\right)^{5 / 2}} .
\end{aligned}
$$

In the above expression appears two contributions which cannot be evaluated analytically. Again if we are inclined to know how these terms behave as a function of the variable $\xi$ we have to proceed a numerical investigation. The explicit expressions writing in terms of $\xi$ are given below:

$$
S_{6}(\xi)=\sum_{n=1}^{\infty}(-1)^{n} \frac{\operatorname{arctanh}\left(\frac{1}{\sqrt{1+\mathrm{n}^{2} \xi^{2} / 16}}\right)}{\left(1+n^{2} \xi^{2} / 16\right)^{3 / 2}}
$$

and

$$
S_{7}(\xi)=\sum_{n=1}^{\infty}(-1)^{n} \frac{\operatorname{arctanh}\left(\frac{1}{\sqrt{1+\mathrm{n}^{2} \xi^{2} / 16}}\right)}{\left(1+n^{2} \xi^{2} / 16\right)^{5 / 2}} .
$$

From our numerical analysis, developed in the high-temperature regime, we obtained the following results:

$$
S_{6}(\xi)=\frac{a_{6}}{\xi^{q_{6}}}
$$

and

$$
S_{7}(\xi)=\frac{a_{7}}{\xi^{q_{7}}},
$$

where $a_{6}=-0.552$ with $q_{6}=0.184 \pm 0.019$ and $a_{7}=-0.552$ with $q_{7}=$ $0.184 \pm 0.019$. So we can conclude that these two terms present the same order of correction to $\left\langle\bar{T}_{00}\right\rangle_{\beta}$ and are not relevant. So the dominant contribution comes from the term independent of the distance from the point to the global monopole, followed by a term of order $1 / r^{2} \beta^{2}$.

For the case $\alpha=1 / 3$ the procedure is similar to this previous one. The integration in (46) can be done and after some intermediate steps it is possible to conclude that the dominant contribution for the energy density, in hightemperature limit is also given by

$$
\left\langle\bar{T}_{00}(x)\right\rangle_{\beta}=\frac{7 \pi^{2}}{120 \beta^{4}}+O\left(\frac{1}{r^{2} \beta^{2}}\right) .
$$


The same dominant behavior is obtained for the case $\alpha=1 / 4$. We shall not repeat the intermediate steps.

As our final comment in this appendix, we want to say that the dominant contributions for $\left\langle\bar{T}_{00}(x)\right\rangle_{\beta}$ in these three examples, coincides with the result found for a flat spacetime, $\left\langle\bar{T}_{00}(x)\right\rangle_{\beta}=\frac{7 \pi^{2}}{120 \beta^{4}}$. However, sub-dominant contributions are consequence of the non flat geometry of this spacetime. 


\section{References}

[1] E. R. Bezerra de Mello, V. B. Bezerra and N. R. Khusnutdinov, Phys. Rev. D 60, 063506 (1999).

[2] M. Barriola and A. Vilenkin, Phys. Rev. Lett. 63, 341 (1989).

[3] L. Dolan L and R. Jackiw, Phys. Rev. D 9, 3320 (1974).

[4] J. D. Bjorken and S. D. Drell, Relativistic Quantum Mechanics (McGraw-Hill, New York, 1964).

[5] I. S. Gradshteyn and I. M. Ryzhik, Table of Integrals, Series and Products, Academic Press, Inc. 1980 .

[6] H. W. Braden, Phys. Rev. D 25, 1028 (1982).

[7] N. D. Birrell and P. C. W. Davis, Quantum Fields in Curved Space (Cambridge University Press, Cambridge, England, 1982).

[8] R. M. Wald, Commun. Math. Phys. 54, 1 (1977).

[9] S. M. Christensen and M. J. Duff, Phys. Lett. 76B, 571 (1986).

[10] S. M. Christensen, Phys. Rev. D 17, 946 (1978).

[11] B. Linet, Class. and Quantum Gravity, 13, 97 (1996).

[12] F. C. Cabral and E. R. Bezerra de Mello, Class. Quantum Grav. 18, 1637 (2001). 


\section{B Figure Captions}

Figure 1: The figures 1(a) and 1(b) exhibit, respectively, the behavior of $S_{1}$ and $S_{2}$ with $\xi$ in the region $[0.01 ; 0.1]$.

Figure 2: This figure exhibits the logarithmic behavior for both functions, $S_{1}$ and $S_{2}$ with the logarithm of $\xi$, in the region [0.01;0.1]. From it, it is possible to estimate the leading dependence for both quantities with $\xi$.

Figure 3: The figure 3(a) exhibits the behavior of $S_{3}$ with $\xi$ in the region $[0.01 ; 0.1]$. The figure $3(\mathrm{~b})$ exhibits the logarithmic behavior for $S_{3}$ with the logarithm of $\xi$, in the region $[0.01 ; 0.1]$. From it, it is possible to estimate its leading dependence with $\xi$.

Table 1: The table 1, exhibits the exponents $q_{4}$ with their respective coefficients $a_{4}$ of $S_{4}$ for different values of $\alpha$.

Table 2: The table 2, exhibits the exponents $q_{5}$ with their respective coefficients $a_{5}$ of $S_{5}$ for different values of $\alpha$. 

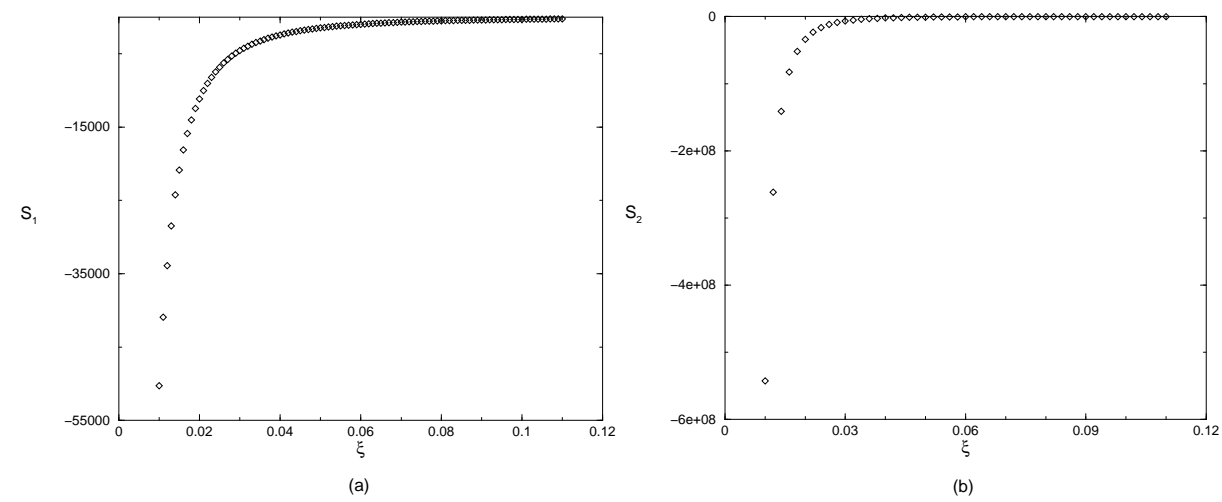

Figure 1:

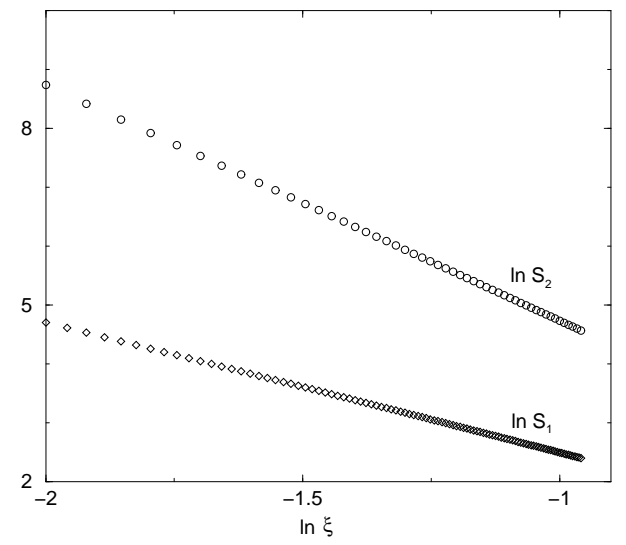

Figure 2: 

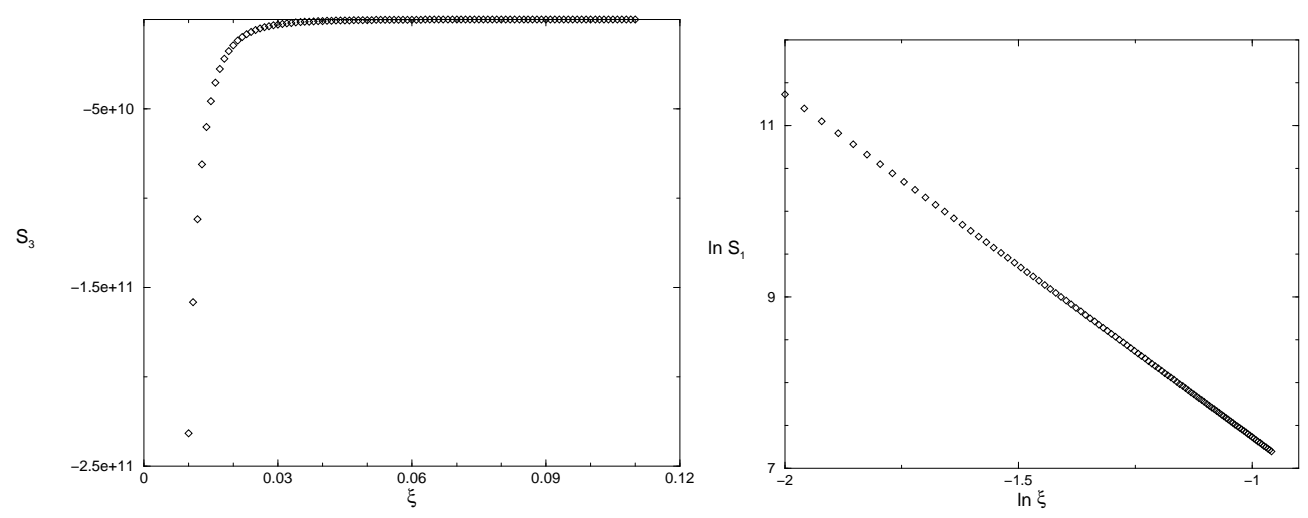

Figure 3:

\begin{tabular}{||c|c|c||}
\hline \hline \multicolumn{3}{|c|}{ Table 1} \\
\hline$\alpha$ & \multicolumn{1}{|c||}{$q_{4}$} & $a_{4}$ \\
\hline 1000 & $2.930375608 \pm 0.1669581160$ & -2.596702947 \\
\hline 1500 & $2.8414692776 \pm 0.1335029194$ & -2.348446259 \\
\hline 2000 & $2.788466901 \pm 0.1156906043$ & -2.193442147 \\
\hline 2500 & $2.751914554 \pm 0.1042923505$ & -2.082895068 \\
\hline 3000 & $2.724562303 \pm 0.09622178978$ & -1.997972468 \\
\hline \hline
\end{tabular}

Table 1:

\begin{tabular}{||c|c|c||}
\hline \hline \multicolumn{3}{|c||}{ Table 2} \\
\hline$\alpha$ & $q_{5}$ & $a_{5}$ \\
\hline 1000 & $2.632887909 \pm 0.12000918880$ & -3.059963397 \\
\hline 1500 & $2.569103696 \pm 0.09581311868$ & -2.887785837 \\
\hline 2000 & $2.531144121 \pm 0.08291154623$ & -2.780842812 \\
\hline 2500 & $2.505004736 \pm 0.07464879774$ & -2.704862735 \\
\hline 3000 & $2.485469694 \pm 0.06879530831$ & -2.646669348 \\
\hline \hline
\end{tabular}

Table 2: 